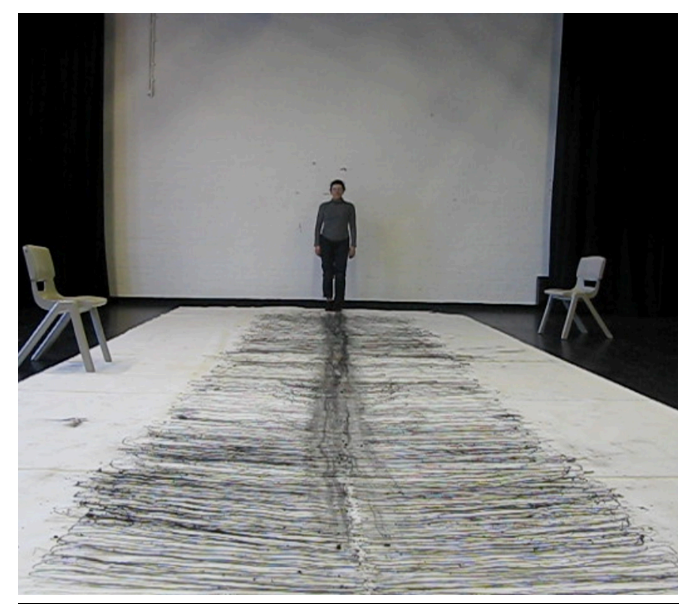

1 Unfolding spine
She's only doing this: scores

is a series of studio-based performance scores, working with iterative task-based constraints. The scores explore drawing as choreographic activity and lining (body drawing line and surface receiving line) in relation to material, surface dimension and gravitational force. Score operates as task, event and document.

\section{Unfolding spine}

Lining paper, scoring breath

The body works on the knees low to the horizontal surface, breathing and drawing while retreating backwards over the paper sheet. The lines that appear and spread over the paper sheet on either side of a central spinal pathway form a diagram, making evident the bilateral symmetry of the arm movements from the spine. As lining and breathing work in sync in real-time, line is breathed and breath is scored.

\section{Parallel=lines \\ leading line, re-tracing line}

Standing facing wall - at eye-level draw a line away - choose where to take line and when to stop - draw a second line returning to its start - repeat the process starting and ending at this place - always a free line a way - a parallel line back. Attend only to and stay close to point of contact of chalk and wall - when meeting an existing line - break contact and continue on other side of existing line - continue process

This score was performed alone in the studio and documented over 40 minutes. I then washed the wall with water and left. 


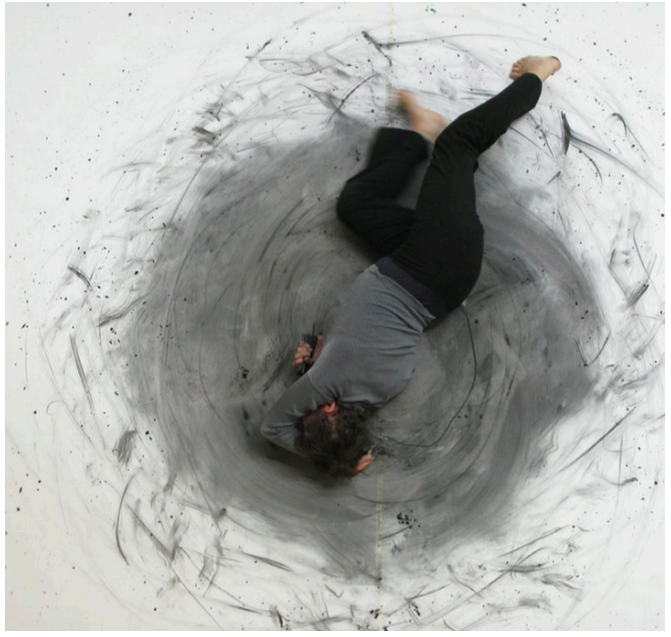

$3 \mathrm{Em}[\mathrm{bed}\}$ ding circle
3 Em[bed]ding circle

Touching surfaces, scoring time

As a body circles and draws, charcoal lines appear on the paper to be wiped over an instance later by the circling body. In duration and repetition of circling, lining, wiping and overlay[er]ing of line, a circle-ness becomes evident on the paper and expands in scale over the surface. Peripheral debris lies scattered over the surface and charcoal dust rises into the air.

She touches paper, holds charcoal, feels pressure. She exerts, tenses, pushes, yields. In slowly stilling her body, she notices miniscule shifts and drops of weight and touch, and sounds of breath, charcoal, paper in an upcloseness to the material surface. She observes the quietness and low space she inhabits. 
